\title{
Jun and JunD-dependent functions in cell proliferation and stress response
}

\author{
A Meixner ${ }^{1}$, F Karreth ${ }^{2}$, L Kenner ${ }^{3,4}$, JM Penninger ${ }^{1}$ and EF Wagner ${ }^{*, 5}$
}

Jun is essential for fetal development, as fetuses lacking Jun die at mid-gestation with multiple cellular defects in liver and heart. Embryos expressing JunD in place of Jun (Jun ${ }^{\mathrm{d} / \mathrm{d}}$ ) can develop to term with normal fetal livers, but display cardiac defects as observed in fetuses lacking Jun. Jun ${ }^{\mathrm{d} / \mathrm{d}}$ mouse embryonic fibroblasts (MEFs) exhibit early senescence, which can be rescued by EGF and HB-EGF stimulation, probably through activation of Akt signaling. Thus, JunD cannot functionally replace Jun in regulating fibroblast proliferation. In $\mathrm{Jun}^{-1-}$ fetal livers, increased hydrogen peroxide levels are detected and expression of $\mathrm{Nrf1}$ and $\mathrm{Nrf2}$ (nuclear erythroid 2-related transcription factors) is downregulated. Importantly, increased oxidative stress as well as expression of Nrf1 and Nrf2 is rescued by JunD in Jun ${ }^{\mathrm{d} / \mathrm{d}}$ fetal livers. These data show that Jun is of critical importance for cellular protection against oxidative stress in fetal livers and fibroblasts, and Jun-dependent cellular senescence can be restored by activation of the epidermal growth factor receptor pathway.

Cell Death and Differentiation (2010) 17, 1409-1419; doi:10.1038/cdd.2010.22; published online 19 March 2010

The Jun members - Jun, JunB, and JunD - belong to a family of related molecules, which together with the Fos proteins (Fos, FosB, Fra1, and Fra2) and members of the ATF and CREB families dimerize to form AP- 1 complexes. ${ }^{1}$ As a result, specific AP-1 complexes activate or repress transcriptional responses to control cell proliferation, differentiation, and death. $^{2}$

Jun-deficient embryos die at embryonic day 12.5 (E12.5) with abnormalities in liver and heart. ${ }^{3,4}$ Hepatocyte-specific deletion of Jun in adult livers affects cell survival and cell-cycle progression ${ }^{5}$ and Jun cooperates with carcinogens to induce hepatocellular carcinomas. ${ }^{6}$ Impaired p21-dependent liver regeneration was shown in livers lacking Jun. ${ }^{7}$ Furthermore, mice lacking Jun in hepatocytes display increased hepatotoxicity on concanavalin A treatment. ${ }^{8}$ This is likely caused by increased oxidative stress, as it can be rescued pharmacologically by nitric oxide delivery to the liver.

Mice lacking JunD are viable ${ }^{9}$ but show enhanced cardiomyocyte apoptosis and fibrosis, ${ }^{10}$ develop chronic kidney disease, ${ }^{11}$ and exhibit increased bone formation. ${ }^{12}$ JunD regulates genes involved in the antioxidant defense and hydrogen peroxide $\left(\mathrm{H}_{2} \mathrm{O}_{2}\right)$ production, and exhibits angiogenesis by controlling VEGF transcription. ${ }^{13}$ Furthermore, an important function for JunD in metabolism and oxidative stress was described, as JunD can modulate insulin/insulinlike-growth factor 1 signaling and longevity. ${ }^{14}$
Mice expressing a variant of Jun, in which the Jun $\mathrm{N}$-terminal kinases (JNK) phosphoacceptor sites were mutated to alanines (Jun $\left.{ }^{A A}\right)$, are viable, although they are smaller than wild-type littermates. ${ }^{15}$ Mice having Jun replaced by JunB develop to birth, indicating that JunB can substitute for Jun during embryonic development. ${ }^{16}$ On the other hand, JunB-deficient embryos die at E8.5-10.5 because of multiple defects in extra-embryonic tissues. ${ }^{17}$ Moreover, JunB was found to be a critical player in endothelial cell morphogenesis by regulating expression of core-binding factor $C B F \beta$ and $V E G F$ suggesting that embryonic lethality of JunBdeficient embryos is likely because of a failure in hypoxiamediated placentation and angiogenesis. ${ }^{18,19}$

Jun is able to suppress p53 gene transcription, and $\mathrm{Jun}^{-/-}$ mouse embryonic fibroblasts (MEFs) have increased expression of $\mathrm{p} 53,{ }^{20}$ a severe proliferation defect, undergo premature senescence, and are sensitive to p53-induced growth arrest on UV irradiation. ${ }^{4,20-22}$ JunD also interacts with the p53 pathway as Jund ${ }^{-1}$ MEFs exhibit a proliferation defect, which is less severe than that of $\mathrm{Jun}^{-/-}$cells and is caused likely by increased $p 19 A R F$ expression. ${ }^{23}$ Jun also stimulated cell-cycle progression through induction of cyclin $D 1$ transcription $^{24}$ and expression of cyclin D1 is downregulated in $\mathrm{Jun}^{-1-}$ MEFs. ${ }^{20,21}$ Immortalized Jund $^{-1-}$ cells show increased proliferation likely as a consequence of increased cyclin D1 expression. ${ }^{23}$ Overexpression of JunD in immortalized

\footnotetext{
${ }^{1}$ Institute of Molecular Biotechnology of the Austrian Academy of Sciences (IMBA), Dr. Bohr-Gasse 3, Vienna A-1030, Austria; ${ }^{2}$ Cancer Research UK Cambridge Research Institut, Li Ka Shing Centre, Robinson Way, Cambridge CB2 ORE, UK; ${ }^{3}$ Ludwig Boltzmann Institute for Cancer Research (LBI-CR), Währingerstraße 13a, Vienna A-1090, Austria; ${ }^{4}$ Medical University, Institute of Clinical Pathology, Währingergürtel 18-20, Vienna A-1090, Austria and ${ }^{5}$ Banco Bilbao Vizcaya Argentaria (BBVA)-Foundation, Cancer Cell Biology Programme, Centro Nacional de Investigaciones Oncológicas (CNIO), Melchor Fernández Almagro 3, Madrid E-28029, Spain ${ }^{*}$ Corresponding author: EF Wagner, Cancer Cell Biology Programme, Centro Nacional de Investigaciones Oncológicas (CNIO), Melchor Fernández Almagro 3, Madrid E-28029, Spain. Tel: + 34917328 000; Fax: + 34912246 980; E-mail: ewagner@cnio.es

Keywords: Jun/AP-1 substitution; development; MEFs; fetal liver; EGFR signaling; oxidative stress

Abbreviations: BHA, butylated hydroxyanisole; ChIP, chromatin immunoprecipitation; EGFR, epidermal growth factor receptor; ERK, extracellular signal-regulated kinases; FCS, fetal calf serum; GCLC, glutamate-cysteine ligase catalytic subunit; GCLM, glutamate-cysteine ligase regulatory subunit; GSS, glutathione synthetase; $\mathrm{H}_{2} \mathrm{O}_{2}$, hydrogen peroxide; HPRT, hypoxanthine-guanine phosophoribosyltransferase; JNK, Jun N-terminal kinase; MEFs, mouse embryonic fibroblasts; NAC, $N$-acetylL-cysteine; Nrf, nuclear erythroid-related transcription factors

Received 13.7.09; revised 17.12.09; accepted 14.1.10; Edited by P Bouillet; published online 19.3.10
} 
3T3 cells and T cells resulted in reduced proliferation suggesting that JunD is a negative regulator of proliferation. ${ }^{25,26}$

The nuclear erythroid-related transcription factors (Nrf) are important regulators of cellular redox homeostasis through their capacity to induce the expression of reactive oxygen species detoxification enzymes and other antioxidant proteins. Similar to Jun-deficient embryos, Nrf1 null embryos die around E13.5 of embryonic development and display small livers, probably because of the failure in antioxidant gene expression and increased apoptosis. ${ }^{27}$ Moreover, embryonic stem (ES) cells deficient in Nrf1 do not contribute to adult livers in chimeras. ${ }^{27}$ In contrast, Nrf2 - similar to JunD - is dispensable for mouse development, ${ }^{28}$ and Nrf2 is involved in G2/M cell-cycle block and controls Akt activity. ${ }^{29}$ Adult livers lacking Nrf2 display reduced Jun levels ${ }^{30}$ and exhibit impaired liver regeneration and oxidative stress. ${ }^{31}$ Furthermore, Nrf2 has a function in age-related diseases, such as neurodegeneration, chronic inflammation, and cancer. ${ }^{32}$ Interestingly, fibroblast proliferation was not affected neither by the loss of Nrf1 nor Nrf2, ${ }^{27}$ although double mutant Nrf1-Nrf2 MEFs enter early senescence, possibly because of increased p53 levels and severe oxidative stress. ${ }^{27}$

Here, we show that JunD can substitute for Jun during fetal development, although embryos display cardiac defects similar to fetuses lacking Jun. Increased $\mathrm{H}_{2} \mathrm{O}_{2}$ production and downregulation of $\mathrm{Nrf1}$ and Nrf2 expression of Jundeficient fetal livers was also rescued by JunD. It seems that Jun and JunD can protect cells and tissues by regulating detoxification and antioxidant genes as was reported for Nrf1/Nrf2. Jun ${ }^{\mathrm{d} / \mathrm{d}}$ MEFs displayed impaired proliferation, early senescence, and impaired Akt signaling, reminiscent of cells lacking Jun. Aberrant proliferation and senescence of Jun ${ }^{d / d}$ MEFs was abolished by high levels of EGF or HB-EGF, but these treatments had no effect on cells lacking Jun. Thus, in contrast to JunB, JunD cannot functionally replace Jun in fibroblast proliferation, but has the potential to rescue mouse development to birth.

\section{Results}

Generation of JunD knock-in mice. A Jun JunD $\left(J u n^{\mathrm{d} /+}\right)$ knock-in allele was generated to disrupt expression of the endogenous Jun gene allowing simultaneous expression of the complete coding region of JunD. Correctly targeted ES cell clones were identified by PCR and verified by Southern blot analysis (Figure 1a and b). Heterozygous offspring were crossed to a general deleter-Cre to remove the floxed PGK $\beta$ geo selection cassette (Figure 1a), which encodes a neolacZ fusion protein. Heterozygous $\mathrm{Jun}^{\mathrm{d} /+}$ offspring were intercrossed to obtain homozygous Jun ${ }^{\mathrm{d} / \mathrm{d}}$ embryos (Figure 1c).

To test whether the described targeting event replaced Jun with the JunD coding region, Jun ${ }^{\mathrm{d} /+}$ mice were crossed with Jund $^{-1-}$ mice. Analysis of 3-week-old Jun ${ }^{\mathrm{d} /}+\mathrm{Jund}^{-1-}$ mouse tissues and various organs, prepared from E12.5 embryos, showed Jund expression in which Jun is normally expressed (Figure 1d and data not shown). There was no change in Junb expression. Thus, the JunD knock-in allele is expressed with a pattern similar to the endogenous Jun gene.

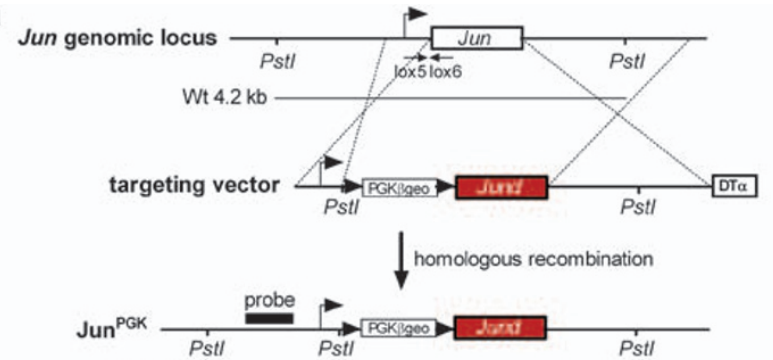

h. r. $2.8 \mathrm{~kb}$
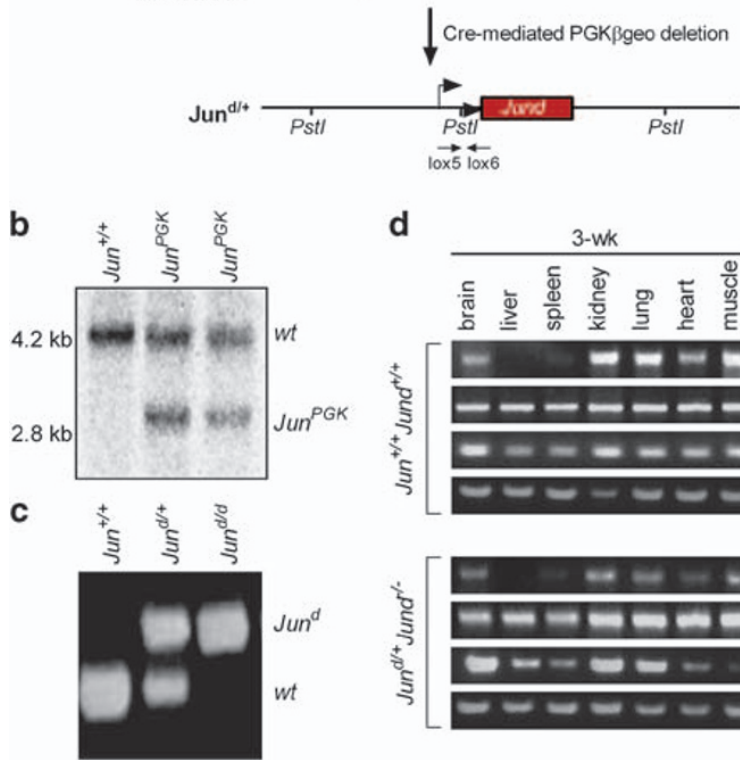

d
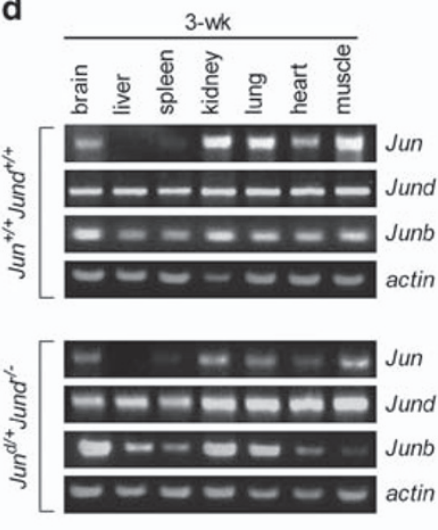

Figure 1 Generation of Jun ${ }^{d / d}$ knock-in mice. (a) Schematic drawing of the targeting strategy used to insert a Jund gene into the Jun locus. Jun coding sequences are indicated by a white box, JunD coding sequences by a red box. Homologous recombination between the dashed lines generates the mutant $J u n^{\text {PGK } \beta g e o: d /+}$ allele. Using the TRAP-cre deleter strain, heterozygous Jun ${ }^{\mathrm{d} /+}$ mice were generated by germline deletion of the floxed PGK $\beta$ geo allele. DT $\alpha$ corresponds to diphtheria toxin $\alpha$, the probe used for Southern blot is shown by a black box and loxP sites are depicted by triangles. (b) Southern blot analysis of Jun ${ }^{\mathrm{PGK}} \beta^{\text {geo:d/ }}+$ (Jun $^{\mathrm{PGK}}$ ) ES cell genomic DNA digested with Pstl. Genotypes and positions of the expected bands are indicated. (c) Genotype analysis of progeny from a heterozygous $\left(J u n^{d /+}\right.$ ) intercross. PCR analysis was performed by using primers lox5 and lox6 shown in (a). Genotypes and positions of the expected wildtype (wt; $380 \mathrm{bp}$ ) and mutant (Jun ${ }^{\mathrm{d}} ; 440 \mathrm{bp}$ ) bands are indicated. (d) Expression pattern of Jun, Jund, and Junb mRNAs isolated from Jun ${ }^{+1+}$ Jund $^{+1+}$ and Jun $^{\mathrm{d} /}+$ Jund $^{-l-}$ 3-week mouse tissues by RT-PCR analysis. Actin serves as a loading control

JunD partially rescues the embryonic lethality of Jundeficient embryos. A total of $35 \%$ of homozygous $J u n^{\mathrm{d} / \mathrm{d}}$ mice were born (Figure 2a; Table $1 \mathrm{~A}$ ). The remaining $65 \%$ died between E12.5 and E13.5 because of defects in the neural tube region and/or a complete delay of development (Figure 2b; Table $1 \mathrm{~A}$ and data not shown). Jun ${ }^{\mathrm{d} / \mathrm{d}}$ newborns displayed open eyes (Figure $2 \mathrm{a}$ and $\mathrm{c}$ ) and died within the first hours after birth. Thus, JunD with limited efficiency can rescue $\mathrm{Jun}^{-1-}$ embryonic lethality up to birth.

Histological analysis of E18.5 embryos and newborns showed that the separation of the cardiac outflow tract into aorta and pulmonary artery had not occurred (Figure 2d, E18.5 and data not shown). This persistence of the heart 

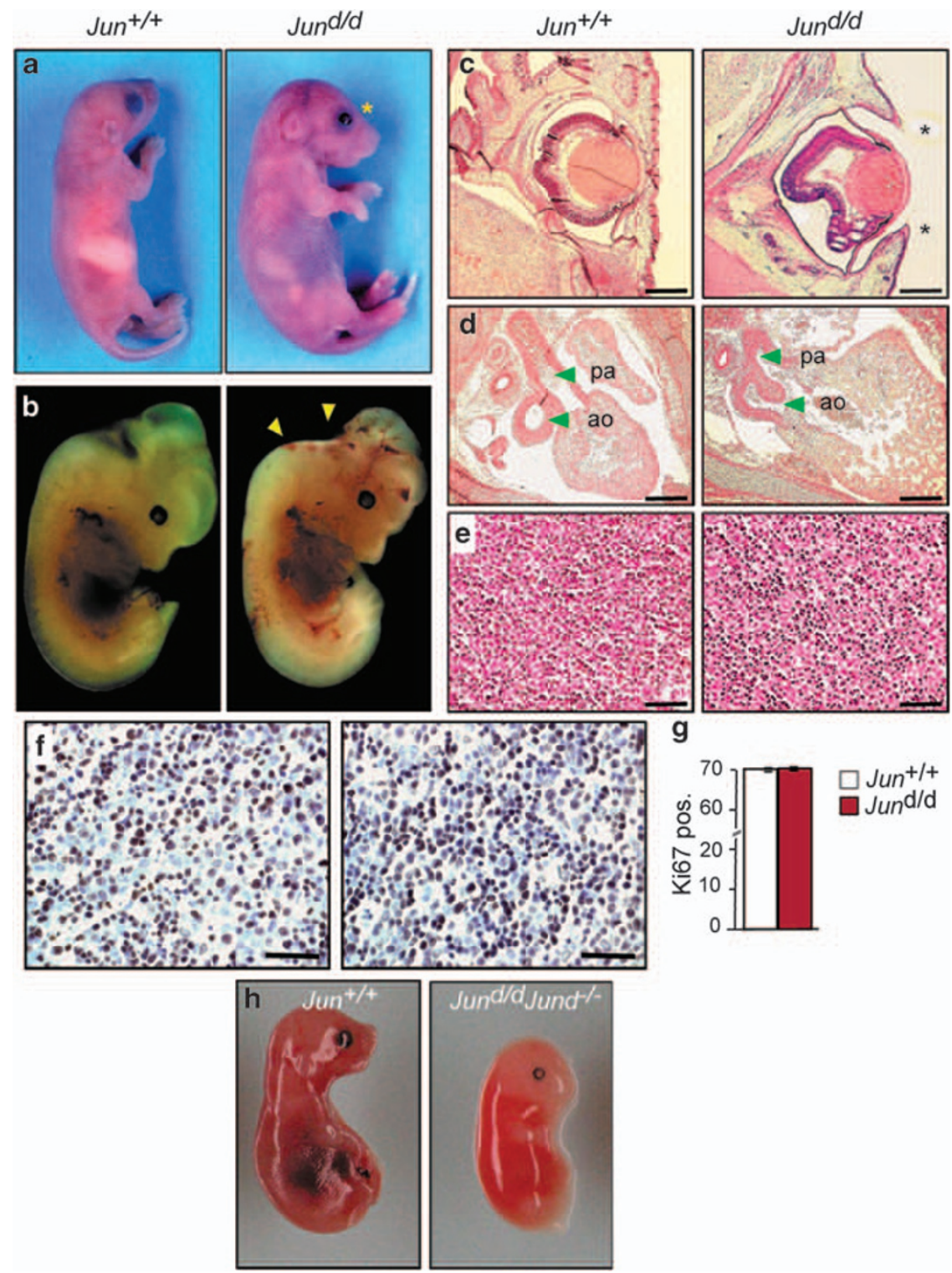

Figure 2 Histological analyses of Jun ${ }^{\mathrm{d} / \mathrm{d}}$ embryos and genetic complementation with Jund ${ }^{-1-}$ mice. (a) Phenotypic appearance of a newborn wild-type (Jun ${ }^{+/+}$) and $J u n^{d / d}$ littermate. The open eyes are indicated by an asterisk. (b) Jun ${ }^{+/+}$E12.5 embryo (left) and Jun ${ }^{\mathrm{d} / \mathrm{d}}$ mutant embryo (right) with neural tube closure defects indicated with arrowheads at E12.5. (c) Histological sections of E18.5 embryos showing the absence of eyelid fusion (asterisks) in Jun ${ }^{\text {d/d }}$ embryos. Scale bar, $400 \mu \mathrm{m}$. (d) Normal right ventricular outflow tract and aorta (ao; arrows) at E18.5. The outflow in the Jun ${ }^{\mathrm{d} / \mathrm{d}}$ embryos tract forms a single vessel (truncus arteriosus communis), before dividing into aorta and pulmonary artery (pa; arrows). Scale bar, $400 \mu \mathrm{m}$. (e) Liver sections of E18.5 Jun ${ }^{+/+}$and Jun ${ }^{\mathrm{d} / \mathrm{d}}$ littermates. Scale bar, $100 \mu \mathrm{m}$. (f) Liver sections of E12.5 Jun ${ }^{+/+}$and $J_{u} n^{d / d}$ littermates. Scale bar, $50 \mu \mathrm{m}$. (g) Quantification of Ki67-positive cells in Jun ${ }^{+/+}$and Jun ${ }^{\text {d/d }}$ liver sections. (h) Macroscopic analysis of an E15.5 Jun ${ }^{d / d} J_{u n d}{ }^{-1-}$ and littermate embryo

defect resembling 'persistent truncus arteriosus' likely contribute to the early postnatal lethality of newborns. Jun ${ }^{\mathrm{d} / \mathrm{d}}$ embryos displayed normal liver architecture throughout development (Figure 2e and data not shown). In agreement with normal liver pathology of $J u n^{\mathrm{d} / \mathrm{d}}$ embryos, there was no difference in proliferation and apoptosis of wild-type and $J u n^{\mathrm{d} / \mathrm{d}}$ E12.5 fetal liver cells (Figure $2 f$ and data not shown). Ki67 staining revealed comparable numbers of proliferating cells in wild-type and Jun ${ }^{\mathrm{d} / \mathrm{d}}$ fetal livers at E12.5 (Figure 2g). However, increased gene dosage of JunD might be crucial for developmental phenotypes and lethality of $J u n^{\mathrm{d} / \mathrm{d}}$ newborns. Indeed,
$J u n^{\mathrm{d} / \mathrm{d}} \mathrm{Jund}^{-1-}$ embryos were severely growth retarded and did not develop beyond E13 of development (Figure 2h; Table 1B). Together, these data indicate that JunD expressed from the Jun locus is not sufficient for normal embryonic development.

Premature senescence of $J u n^{\mathrm{d} / \mathrm{d}}$ MEFs. We next analyzed whether JunD can substitute for Jun in regulating proliferation of MEFs. Expression of JunD in Jun ${ }^{\mathrm{d} / \mathrm{d}}$ MEFs was induced similarly to the endogenous Jun gene (data not shown). Moreover, similar to $\mathrm{Jun}^{-/-}$cells, Jun ${ }^{\mathrm{d} / \mathrm{d}}$ cells 
exhibited growth arrest after two to three passages (Figure 3a). Senescence was further confirmed by intense $\beta$-galactosidase activity in $J u n^{\mathrm{d} / \mathrm{d}}$ MEFs compared with wildtype cells (Figure $3 b$ ). Increased levels of JunD were not the cause of the proliferation defect, as $J u n^{\mathrm{d} / \mathrm{d}}$ cells lacking endogenous JunD (Jun ${ }^{\mathrm{d} / \mathrm{d}} \mathrm{Jund}^{-1-}$ ) also failed to proliferate in vitro and underwent premature senescence (Figure $3 \mathrm{c}$ and d). Thus, JunD cannot substitute for Jun in the regulation of proliferation in MEFs.

JunD was able to replace c-Jun in repressing p53 transcription, as p53-mediated transactivation (Figure $3 e$ ) and p53 mRNA and protein expression were comparable with wild-type MEFs (Figure $3 f$ and g). Analyzing p53-target genes $m d m 2, p 21$, and bax1 on UV induction (40J) did not reveal any difference between wild-type and Jun ${ }^{\mathrm{d} / \mathrm{d}}$ MEFs (Figure $3 \mathrm{~h}$ ). Furthermore, p53 and p21 protein levels were similar on UV treatment indicating that JunD is capable of suppressing p53 transcription (Figure $3 \mathrm{~h}$ ). Jun ${ }^{\mathrm{d} / \mathrm{d}}$ MEFs were analyzed for Cdc2, another Jun-dependent cell-cycle regulator, and expression was found to be rescued to wild-type levels (Figure 3i). A Jun-binding site (PF-1, TGA G TCT) has been described for the mouse $p 53$ promoter. ${ }^{20}$ Chromatin immunoprecipitation (ChIP) experiments showed that both JunD and Jun, but not JunB, efficiently bound the p53 domain in wildtype cells, whereas the binding for Jun was almost undetectable in $J_{u n}{ }^{-1-}$ and Jun ${ }^{d / d}$ MEFs (Figure 3l). Moreover, in addition to decreased cyclin D1 transcription (Figure 3k), the cyclin $D 1$-promoter activity was reduced in exponentially growing
$J u n^{\mathrm{d} / \mathrm{d}}$ cells compared with wild-type MEFs (Figure 3j). Reduced cyclin D1 expression was further confirmed at the protein level in $J u n^{\mathrm{d} / \mathrm{d}}$ cells (Figure $3 \mathrm{k}$ ). In both mouse and human, an AP-1 responsive TRE element (TGT C TCA) has been described in the cyclin D1-promoter proximal region. ${ }^{33} \mathrm{ChIP}$ experiments showed that Jun and JunD bound to the TRE element indicating that Jun and JunD directly control the transcription of cyclin D1 through the TRE-promoter site (Figure $3 \mathrm{~m}$ ).

\section{Reduced epidermal growth factor receptor expression in} $J_{u n}{ }^{\mathrm{d} / d}$ MEFs. Next, we analyzed the function of epidermal growth factor receptor (EGFR), a Jun-target gene having a major function in fibroblast and keratinocyte proliferation. ${ }^{34}$ Expression of EGFR mRNA was downregulated, whereas expression of the ligand $H B-E G F$ or $T G F-\alpha$ was unchanged in $J u n^{\mathrm{d} / \mathrm{d}}$ MEFs (Figure 4a). Reduced EGFR expression was further confirmed at the protein level in $J u n^{\mathrm{d} / \mathrm{d}}$ and $J u n^{-1-}$ MEFs (Figure 4b). When analyzing potential alternative receptors including other EGFRs, we found that ErbB2, $U p a R$, and Igf2R expression was not changed, whereas ErbB3 and ErbB4 expression was not detectable in MEFs (Figure 4c; Supplementary Figure 1A). Addition of EGF or HB-EGF to fibroblast cultures induced proliferation of $J u n^{\mathrm{d} / \mathrm{d}}$ MEFs, but had little effect on Jun ${ }^{-1-}$ MEFs (Figure 4d). In contrast, pharmacological inhibition of the EGFR pathway induced cell death independent of the genotype. Inhibition of EGFR $(20-0.02 \mu \mathrm{m})$, ErbB2 $(20 \mu \mathrm{m})$, and EGFR-ErbB2ErbB4 $(20,2 \mu \mathrm{m})$ had either no effect on proliferation or

Table 1 Genetic analyses of JunD functions in vivo

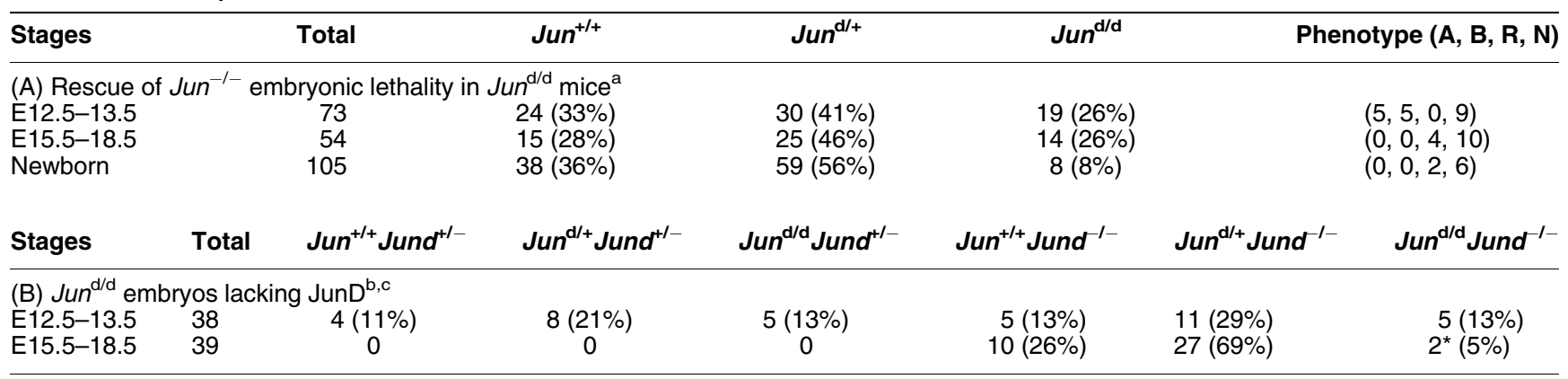

antercrosses between $J u n^{\mathrm{d} /+}$ mice. A, hindbrain exencephaly; R, resorbed embryos; B, delayed development; $\mathrm{N}$, no obvious anomaly; \% $=$ observed frequency.

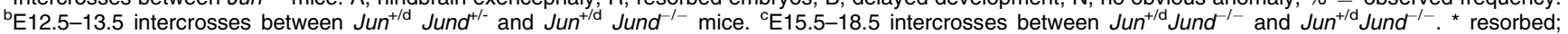
$\%=$ observed frequency.

\footnotetext{
Figure 3 Premature senescence of Jun ${ }^{\mathrm{d} / \mathrm{d}}$ MEFs. (a) Proliferation rates of MEFs. Cumulative cell number of primary cells from Jun ${ }^{+/+}$, Jun ${ }^{\mathrm{d} / \mathrm{d}}$, and Jun ${ }^{-1-}$ cells passaged at 4 day intervals. Arrows indicate the passage number $(n=4)$. (b) $\beta$-galactosidase activity in Jun ${ }^{\mathrm{d} / \mathrm{d}}$ cells that is present only in senescent cells. (c) Proliferation rates of $J_{u n}{ }^{1+}, J_{u n}{ }^{d /+} J_{u n d}{ }^{-1-}$, and Jun ${ }^{\mathrm{d} / \mathrm{d}} J u n d^{-1-}$ MEFs passaged at 4 day intervals and cumulative cell numbers. Arrows indicate the passage number ( $n=3$ ). (d) $\beta$-galactosidase activity is also present in Jun ${ }^{\mathrm{d} / \mathrm{d}} \mathrm{Jund}^{-1-}$ MEFs. (e) Luciferase reporter assays performed in Jun ${ }^{+1+}$, Jun ${ }^{\mathrm{d} / \mathrm{d}}$, and Jun ${ }^{-1-}$ exponentially growing MEFs transfected with the $p 53$-luc-promoter luciferase constructs $(n=3)$. (f) qPCR analysis of $p 53$ expression in exponentially growing MEFs. (g) p53 protein levels in exponentially growing (ex), serum-starved (st), and serum-stimulated (sti) Jun ${ }^{+1+}$, Jun ${ }^{\mathrm{d} / \mathrm{d}}$, and Jun ${ }^{-1-}$ MEFs ( $\left.n=2-4\right)$. Actin serves as loading control. (h) RT-PCR analysis (upper panel) of the indicated genes on UV induction (40J) in Jun ${ }^{+I+}$ and Jun ${ }^{\mathrm{d} / \mathrm{d}}$ MEFs. p53 and p21 protein induction (lower panel) on UV induction (40J) in Jun ${ }^{+1+}$ and Jun ${ }^{\mathrm{d} / \mathrm{d}}$ MEFs $(n=2)$. (i) Cdc2 expression in Jun ${ }^{+l+}$, Jun ${ }^{\mathrm{d} / \mathrm{d}}$, and Jun ${ }^{-1-}$ passage 4 exponentially growing MEFs. Actin serves as a loading control. (j) Luciferase reporter assays performed in Jun ${ }^{+1+}$, Jun ${ }^{\mathrm{d} / \mathrm{d}}$, and Jun ${ }^{-1-}$ MEFs transfected with the cyclin D1-luc-promoter luciferase construct $(n=3)$. Error bars represent S.D.; asterisk, $P<0.05$. (k) Serum induction of cyclin D1 RNA and protein in serum-starved (stv) and serum-stimulated Jun ${ }^{+I+}$ and Jun ${ }^{\mathrm{d} / \mathrm{d}}$ (upper panel) and Jun ${ }^{+/+}$and Jun ${ }^{-/-}$MEFs (lower panel). Actin expression or actin serves as a loading control $(n=2-3)$. Schematic illustration of the mouse p53 promoter showing the PF1 (TGA C TCT, black circle)-binding site. $^{20}(\mathbf{I})$ and mouse cyclin D1 promoter ( $\left.\mathbf{m}\right)$ showing the TRE element (TGT C TCA, red circle). ${ }^{33}$ Arrows indicate primers amplifying proximal fragments. bp, base pairs. Chromatin of the indicated genotypes was immunoprecipitated with Jun antibodies. End point qPCR-amplified fragments are shown. IgG was used as isotype-specific antibody control. $+1+$, Jun $^{+1+} ;-1-$, Jun $^{-1-} ; \mathrm{d} / \mathrm{d}, \mathrm{Jun}^{\mathrm{d} / \mathrm{d}}$
} 
a

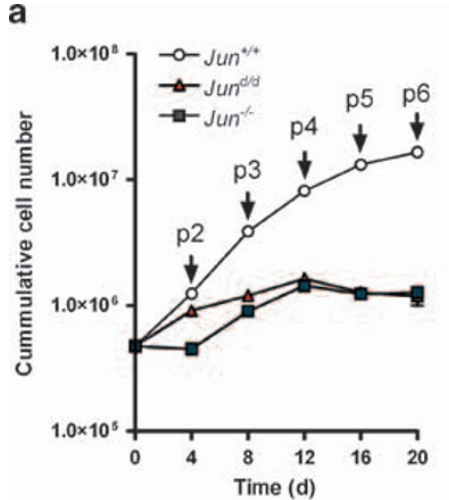

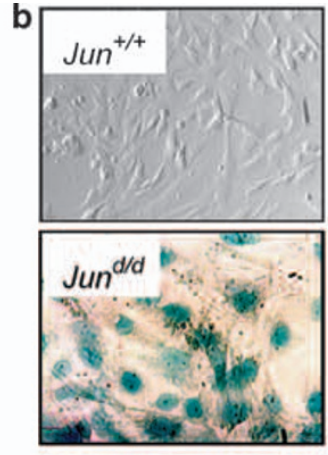

C

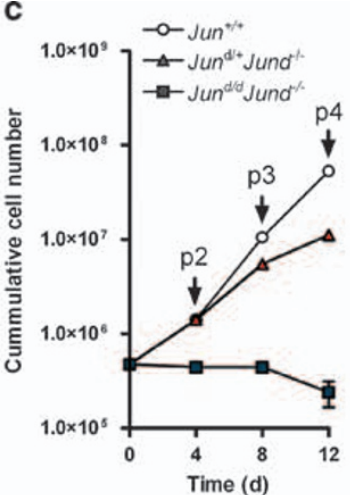

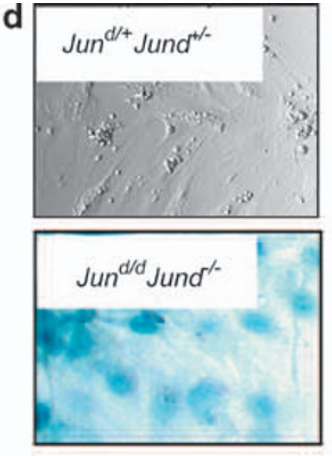

e

f

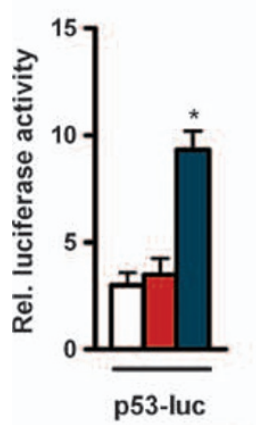

g

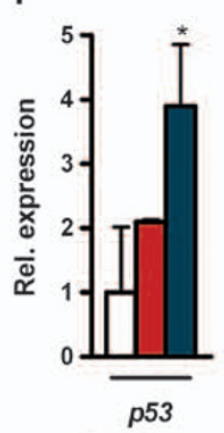

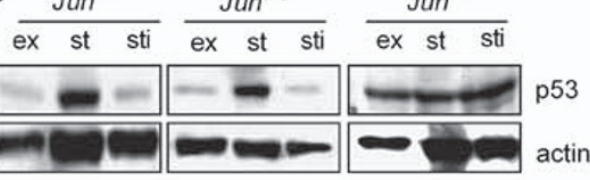

i $\operatorname{Jun}^{+/+} J_{u n}^{d / d} J_{u n}{ }^{-/-}$
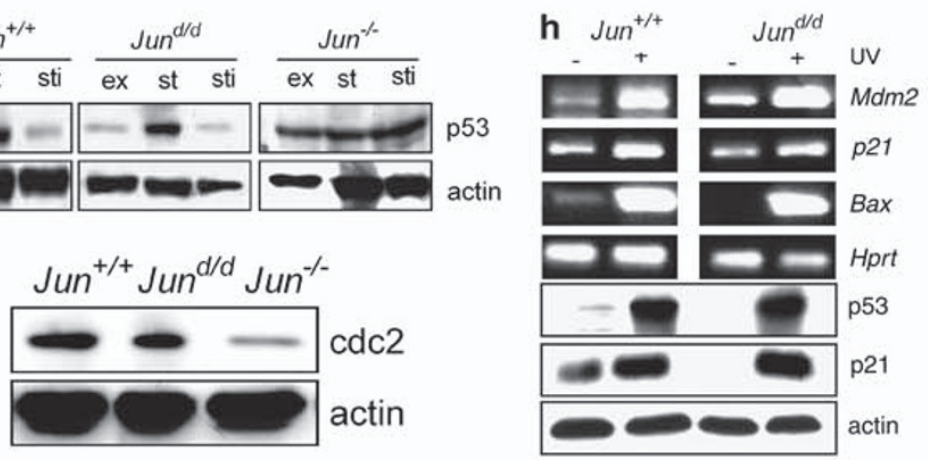

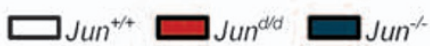

j

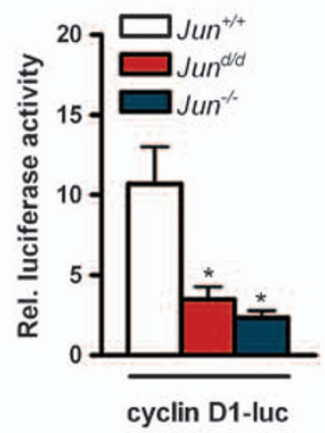

1

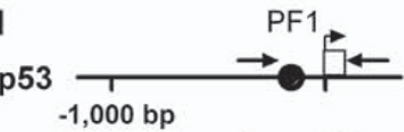

$+/+\quad-/-\quad \mathrm{d} / \mathrm{d}$

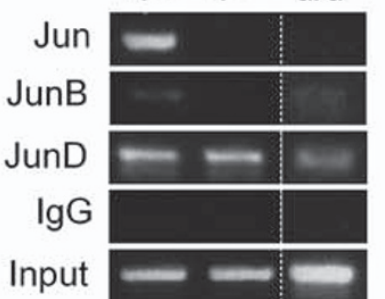

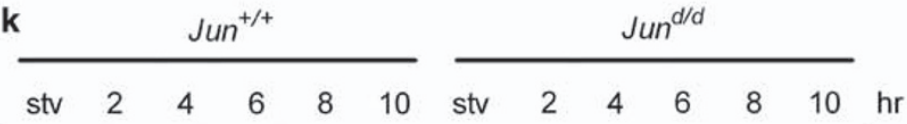
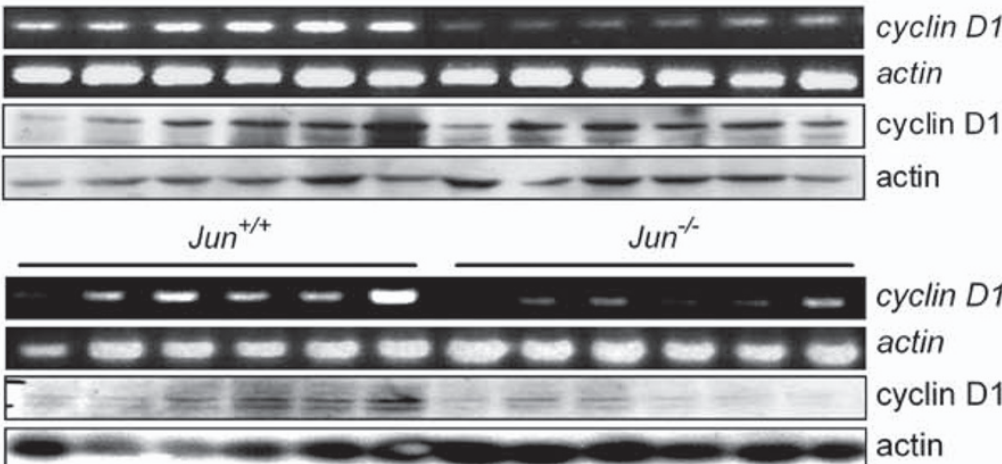

cyclin D1 actin cyclin D1 actin

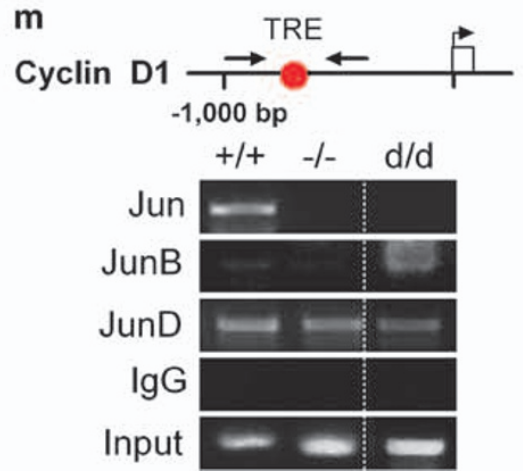


a

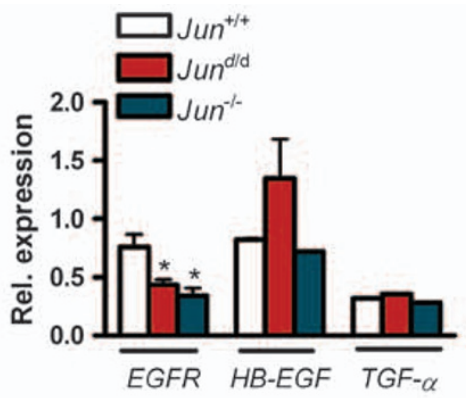

d

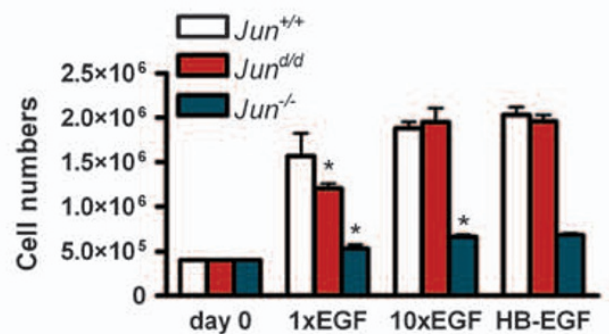

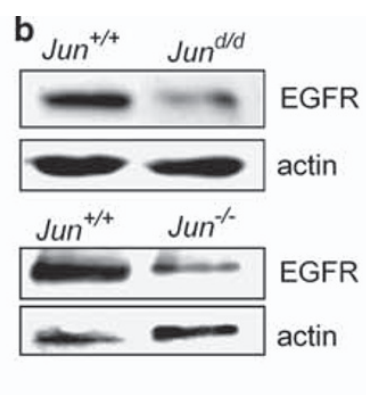

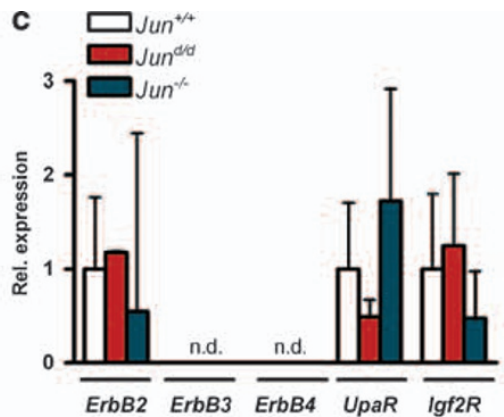

f
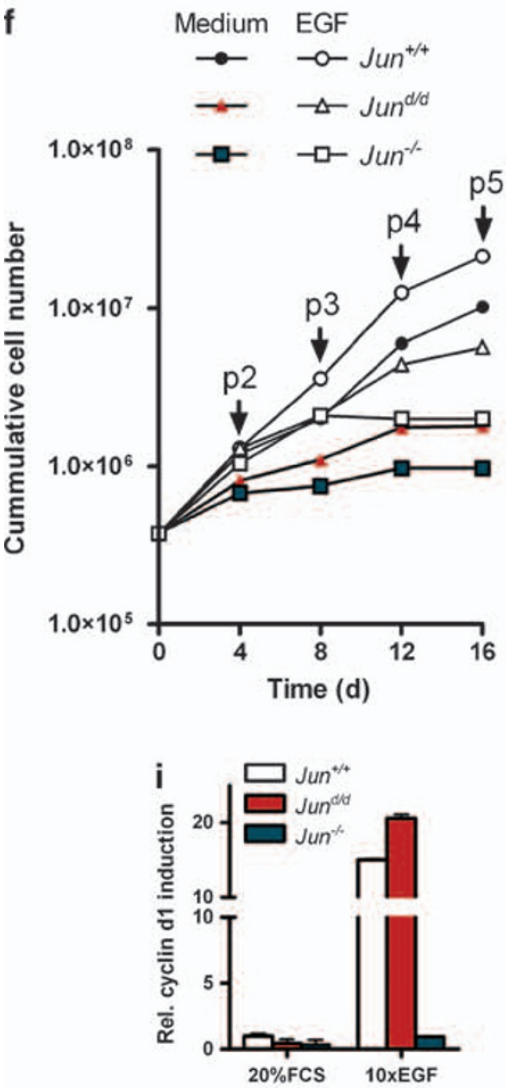

$\frac{\mathrm{g} \mathrm{Jun}^{+/+}}{\operatorname{stv} 25} \frac{J^{2}{ }^{d / d}}{\operatorname{stv} 25} \frac{J^{-/-}}{\operatorname{stv} 25}$

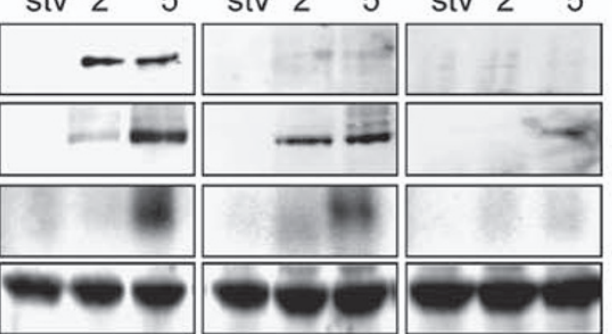

$\min$

p-Tyr1173EGFR

$120 \%$ FCS

p-Tyr1173EGFR

110xEGF

p-Tyr1068EGFR

$110 \times E G F$

actin

h

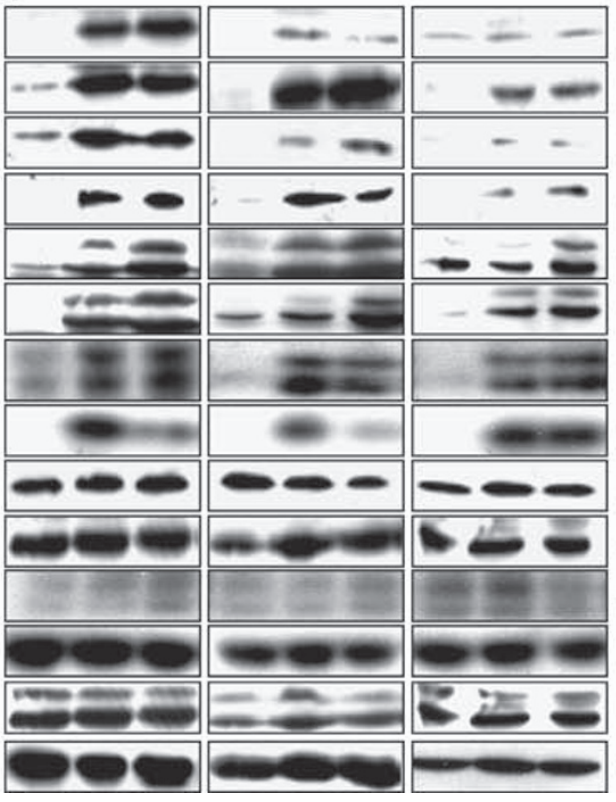

p-Akt/20\%FCS

p-Akt/10xEGF p-GSK-3 $3 / 20 \% F C S$ p-GSK-3ß/10xEGF p-Erk/20\%FCS $\mathrm{p}-J N K / 10 \times E G F$ p-p38/10xEGF Akt

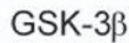
JNK p38 Erk actin p-Erk/10xEGF 
induced cell death on primary or immortalized $\mathrm{MEFs}^{22}$ (Figure 4e; Supplementary Figure $1 \mathrm{~B}$ and $\mathrm{C}$ and data not shown) suggesting that proliferation of MEFs is not affected by EGFR-ErbB2-ErbB4 inhibition.

Besides the mitogenic effect of EGF on Jun ${ }^{\mathrm{d} / \mathrm{d}}$ MEFs, high doses of EGF also rescued premature senescence of $J u n^{\mathrm{d} / \mathrm{d}}$ cells. During the first two passages, cell proliferation was comparable between $J u n^{+1+}, J u n^{\mathrm{d} / \mathrm{d}}$, and Jun ${ }^{-1-}$ MEFs. At passage $4(d=12), J_{u n^{-1-}}$ cells showed significantly reduced proliferation rates (Figure $4 \mathrm{f}$ ), whereas $J u n^{\mathrm{d} / \mathrm{d}}$ cells proliferated almost similar to wild-type cells in the presence of EGF. This indicates that different molecular targets are responsible for the proliferation defects of these two cell populations. EGFR was not phosphorylated in Jun ${ }^{-1-}$ cells under any condition tested, whereas Jun ${ }^{\mathrm{d} / \mathrm{d}}$ cells displayed phosphorylated EGFR after treatment with 10xEGF at tyrosine 1173 and tyrosine 1068 (100 ng/ml) (Figure 4g). Consistent with defective EGFR phosphorylation, the down-stream kinases Akt and GSK-3 $\beta$ were not phosphorylated by either $20 \%$ fetal calf serum (FCS) or high doses of EGF in Jun ${ }^{-1-}$ cells (Figure 4h). In contrast, induction of Akt and GSK-3 $\beta$ phosphorylation was observed in $J u n^{\mathrm{d} / \mathrm{d}}$ cells after treatment with 10xEGF (Figure 4h). In contrast, ERK $1 / 2$ and JNK $1 / 2$ activation was similar in all cells analyzed (Figure $4 \mathrm{~h}$ and data not shown). Furthermore, phosphorylation of p38 MAPK was found to be moderately increased in Jun ${ }^{-1-}$ MEFs (Figure 4h). Cyclin D1 induction in $J u n^{\mathrm{d} / \mathrm{d}}$ cells on 10xEGF treatment reached similar levels to wild-type MEFs (Figure 4i).

Together, Jun ${ }^{\mathrm{d} / \mathrm{d}}$ MEFs have limited capacity of replication, which is caused by inefficient Jun-dependent gene transcription that can be activated by an Akt-signaling cascade on response to growth factors, such as EGF or HBEGF. The reduction and delay in cyclin $D 1$ expression might contribute to the senescence phenotype, whereas this pathway is not active in the absence of Jun possibly because of upregulated p38 activity.

\section{Increased oxidative stress in $\mathrm{Jun}^{-1-}$ MEFs and fetal} livers is rescued by JunD. JunD was identified to have an important function in regulating oxidative stress by controlling $\mathrm{H}_{2} \mathrm{O}_{2}$ levels. ${ }^{13}$ We next analyzed oxidant toxicity in $\mathrm{Jun} \mathrm{n}^{\mathrm{d} / \mathrm{d}}$ MEFS and fetal liver cells. $\mathrm{H}_{2} \mathrm{O}_{2}$ production was found to be increased in $\mathrm{Jun}^{-1-}$ and $J u \mathrm{n}^{\mathrm{d} / \mathrm{d}}$ MEFs (Figure 5a). Addition of antioxidants, such as ascorbate (vitamin C) or chemical antioxidants such as butylated hydroxyanisole (BHA) and $\mathrm{N}$-acetyl-L-cysteine (NAC) had no effect on cell proliferation of $J u n^{\mathrm{d} / \mathrm{d}}$ and $J u n^{-1-}$ MEFs (Figure $5 \mathrm{~b}$ ).

The expression of Nrf1 and Nrf2 was next analyzed, as these transcription factors are involved in regulating the redox balance. Low levels of Nrf1 and Nrf2 were observed in both $\mathrm{Jun}^{-1-}$ and Jun ${ }^{\mathrm{d} / \mathrm{d}}$ MEFs (Figure 5c) compared with wild-type MEFs. As reported, ${ }^{35}$ low levels of Glutamate-cysteine ligase, catalytic subunit $(\mathrm{Gclc})$ and glutathione (GSH) synthase (Gss), important enzymes responsible for maintaining GSH homeostasis, were found to be expressed at similar levels in $J_{u n}{ }^{\mathrm{d} / \mathrm{d}}$ and $\mathrm{Jun}^{-1-}$ MEFs, although the difference was not significant (Figure 5c). Glutamate-cysteine ligase, modifier subunit (Gclm) and the JunD-target genes Nox4, Cdo1, and Mgst1 were found to be expressed at similar levels in $\mathrm{Jun}^{-1-}$ and $J u n^{\mathrm{d} / \mathrm{d}}$ MEFs (Figure 5c). Interestingly, Nrf1 and Gss expression in $J u n^{d / d}$ cells was almost restored to wild-type levels on EGF treatment, whereas expression was weakly induced in Jun ${ }^{-1-}$ MEFs (Figure $5 d$ and data not shown). Next, we asked whether the defect in $\mathrm{H}_{2} \mathrm{O}_{2}$ production and $\mathrm{Nrf1/2}$ expression is cell-type dependent. Secretion of $\mathrm{H}_{2} \mathrm{O}_{2}$ in $J_{u n}{ }^{\mathrm{d} / \mathrm{d}}$ fetal liver cells was found to be similar to wild-type levels (Figure 6a). However, increased $\mathrm{H}_{2} \mathrm{O}_{2}$ secretion was observed in Jun-deficient fetal liver cells (Figure 6a). Juntarget genes, such as p53 and p21, were undetectable at the protein level in any of the fetal liver extracts analyzed (Figure 6b). Interestingly, expression of Nrf1, Nrf2, Gclc, and Gss was downregulated in Jun ${ }^{-1-}$ fetal liver cells, but rescued by JunD (Figure $6 \mathrm{c}$ ). There was no difference in expression of the JunD-target genes Nox4, Cdo1, and Mgst1 (Figure 6c). Thus, JunD can rescue the oxidative stress response in fetal liver cells lacking Jun, but not in MEFs, suggesting that this phenotype is cell-type specific.

\section{Discussion}

In this study, we show that JunD can partially substitute for Jun-specific functions in embryonic development, such as fetal liver development to birth. Jun-deficient fetal livers secreted high levels of $\mathrm{H}_{2} \mathrm{O}_{2}$, which was rescued by JunD expression in Jun ${ }^{d / d}$ cells. In vitro, JunD was not able to substitute for Jun in cell proliferation of MEFs, and Jun ${ }^{\mathrm{d} / \mathrm{d}}$ cells underwent early senescence, secreted increased $\mathrm{H}_{2} \mathrm{O}_{2}$ levels, expressed reduced levels of EGFR, and exhibited defective EGF-dependent signal transduction. These properties of $J u n^{\mathrm{d} / \mathrm{d}}$ cells are reminiscent of primary MEFs lacking Jun. However, all defects of Jun ${ }^{d / d}$ cells could be overcome by addition of EGF and HB-EGF, whereas $\mathrm{Jun}^{-1-}$ MEFs did not respond to growth factor stimulation. Thus, JunD regulation of specific target genes is strongly dependent on the cellular context (Figure 6d).

When JunD was expressed from the Jun locus, some fetuses developed to birth, showed normal liver architecture, but displayed severe cardiac defects, as earlier described for

Figure 4 Function of EGFR and Akt signaling in Jun ${ }^{\mathrm{d} / \mathrm{d}}$ and $J u n^{-1-}$ MEF proliferation. (a) qPCR measuring EGFR and EGFR ligands (HB-EGF and TGF- $\alpha$ ) in primary $J_{u n}^{+1+}, J u n^{d / d}$, and Jun ${ }^{-1-}$ MEFs $(n=4)$. (b) Western blot of primary MEFs showing reduced expression of EGFR in exponentially growing Jun ${ }^{\mathrm{d} / \mathrm{d}}$ (upper panel) and Jun ${ }^{-/-}$ MEFs (lower panel). (c) ErbB2, ErbB3, ErbB4, UpaR, and Igf2R mRNA in Jun ${ }^{+/+}$, Jun ${ }^{\text {d/d }}$, and $J u n^{-l-}$ MEFs (qPCR, wild-type set to 1, $n=3$ ). n.d., not detectable; (d) Proliferation of MEFs treated with different concentration of EGF or HB-EGF. 1xEGF (10 ng/ml); $10 x E G F(100 \mathrm{ng} / \mathrm{ml})$; HB-EGF $(10 \mathrm{ng} / \mathrm{ml})(n=4)$. (e) MEFs were treated with inhibitors against ErbB2 $(20 \mu \mathrm{M})$, ErbB2-ErbB4-EGFR (2-4-E; $20 \mu \mathrm{M})$, and EGFR $(20 \mu \mathrm{M}, 0.2 \mu \mathrm{M})$ for $48 \mathrm{~h}$ followed by quantification of cell numbers $(n=3)$. (f) Proliferation rates of $J u n^{+/+}$, Jun ${ }^{d / d}$, and $J u n^{-1-}$ MEFs on addition of 10xEGF $(100 \mathrm{ng} / \mathrm{ml})$ passaged at 4 day intervals. Arrows indicate the passage number $(n=4)$. (g) Western blot of primary MEFs showing that EGFR is phosphorylated only on stimulation with $10 x E G F(100 \mathrm{ng} / \mathrm{ml})$ in Jun ${ }^{d / d}$ cells. MEFs were starved for $48 \mathrm{~h}$ in $0.5 \%$ serum and stimulated for the indicated times with $20 \%$ FCS or 10xEGF. (h) Akt, GSK-3 $\beta$, Erk, JNK, and p38 activation in primary MEFs on stimulation as described in (g). (i) Serum-starved MEFs were treated with $20 \% \mathrm{FCS}$ or $10 \mathrm{xEGF}$ for $8 \mathrm{~h}$, followed by qPCR analyses for cyclin $D 1(n=2)$. Error bars represent S.D.; asterisk, $P<0.05$ 


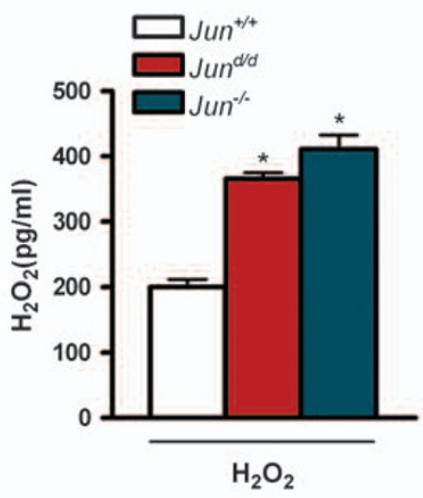

C

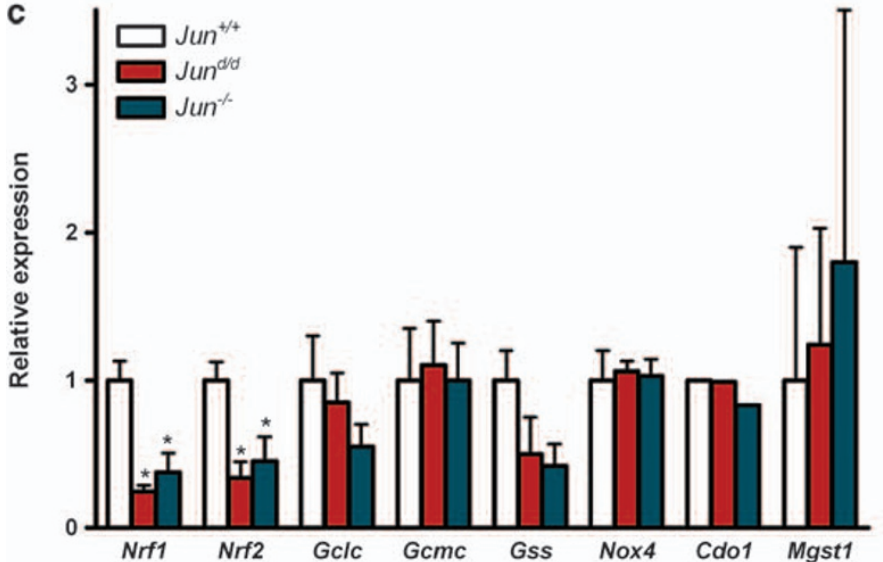

b

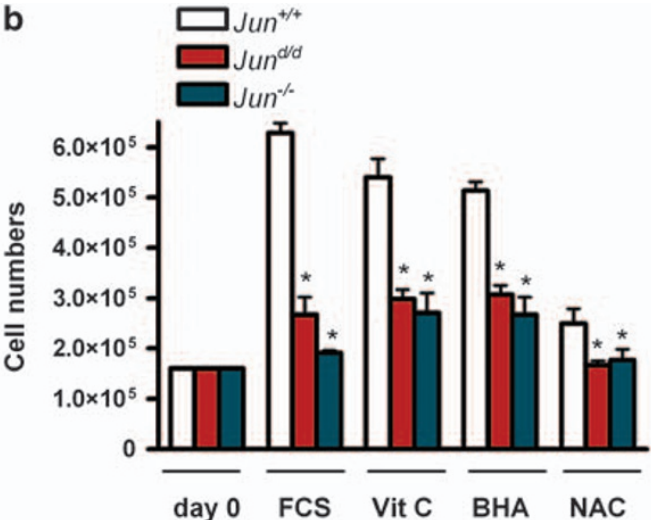

d

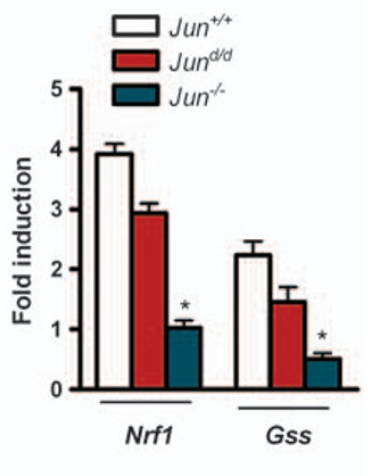

Figure 5 Increased oxidative stress in Jun ${ }^{\mathrm{d} / \mathrm{d}}$ and Jun ${ }^{-/-}$MEFs. (a) Increased secretion of $\mathrm{H}_{2} \mathrm{O}_{2}$ measured by ELISA in exponentially growing Jun ${ }^{\mathrm{d} / \mathrm{d}}$ and Jun ${ }^{-/-}$MEFs $(n=3)$. (b) Proliferation of MEFs in the presence of ascorbate (Vitamin C; $2.5 \mu \mathrm{M})$, BHA $(100 \mu \mathrm{M})$, or $N$-acetyl-cysteine (NAC, 20 mM). Relative cell numbers measured at day 0 and after for 4 days $(n=3)$. (c) JunD does not rescue decreased Nrf1 and Nrf2 expression in Jun ${ }^{-1-}$ MEFs $(n=4)$. The relative mRNA expression of Nrf1/2 and Nrf1/2 targets in primary Jun ${ }^{+1+}$, Jun $^{-l-}$, and Jun ${ }^{d / d}$ MEFs (qPCR, wild-type set to $1, n=4$ ). Error bars represent S.D.; asterisk, $P<0.05$. (d) Serum-starved MEFs were treated with 10xEGF for $8 \mathrm{~h}$, followed by qPCR analyses for Nrf1 and Gss $(n=2)$. Error bars represent S.D.; asterisk, $P<0.05$

a JunB knock-in gene substitution of Jun. ${ }^{16}$ Besides these similarities, several differences were observed. First, all Jun ${ }^{\mathrm{d} / \mathrm{d}}$ newborns died immediately after birth, whereas JunB knockin mice survived until postnatal day 4 . In addition, $50 \%$ of $J_{u n}{ }^{\mathrm{d} / \mathrm{d}}$ embryos died around E12.5 displaying defects in neurulation and growth retardation. However, JunD - similar to JunB - can fully compensate for the lack of Jun in fetal liver development, as impaired proliferation and increased apoptosis of Jun-deficient fetal livers was fully rescued in vivo. Conversely, a functional Jun gene is essential for proper heart development, as neither JunB nor JunD, when expressed from the Jun locus, rescued the heart malformation. In contrast, Jun-deficient mice ectopically expressing a JunB transgene are born without liver and heart defects. ${ }^{16}$ In addition, a JunB transgene is also sufficient to bypass embryonic lethality observed in Junb ${ }^{-/-}$embryos. ${ }^{16,17}$ JunD does not have the ability to rescue both Jun-dependent liver and heart defects in a gene-dosage-dependent manner. Jund transgenic mice ${ }^{26}$ in a Jun ${ }^{-1-}$ background did not develop beyond $E 15.5$, possibly because of different JunD functions in activating/repressing specific target genes (data not shown). This implies that the physiological functions of the structurally similar proteins JunB and JunD partially overlap.
$J u n^{\mathrm{d} / \mathrm{d}}$ MEFs displayed premature senescence and impaired proliferation similar to MEFs lacking Jun. The defect in proliferation was not caused by increased JunD expression, as deletion of endogenous JunD in $J u n^{\mathrm{d} / \mathrm{d}}$ cells also resulted in senescence and in complete cell-cycle block (data not shown). The fact that both JunD and Jun bind to the p53 promoter supports the notion that JunD is capable of suppressing p53 transcription. This is supported by our earlier studies showing that Jun is a major AP-1 component mediating negative regulation of the $p 53$-promoter site PF1. ${ }^{20}$ Importantly, cyclin D1 expression was greatly reduced in $J u n^{\mathrm{d} / \mathrm{d}}$ MEFs compared with wild-type cells. ChIP experiments indicate that apparently all Jun members can influence cyclin $D 1$ transcription by binding to the TRE site in the cyclin D1 promoter. Interestingly, replacement of Jun by JunB rescued the cellular defects associated with loss of Jun, ${ }^{16}$ as these MEFs do not enter premature senescence. Notably, both p16 and cyclin D1 expression was increased in $J u n^{\mathrm{b} / \mathrm{b}}$ cells.

Imbalanced Akt signaling contributed to the resistance of cells to EGF. Indeed, insufficient Akt and GSK-3 $\beta$ phosphorylation on serum stimulation was completely restored in $J u n^{\mathrm{d} / \mathrm{d}}$ cells using increasing EGF concentrations indicating that an 
a

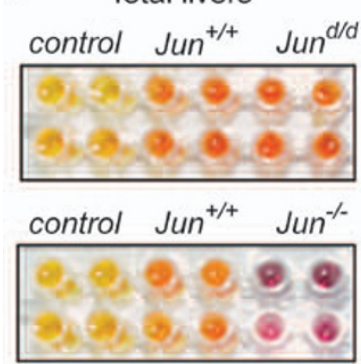

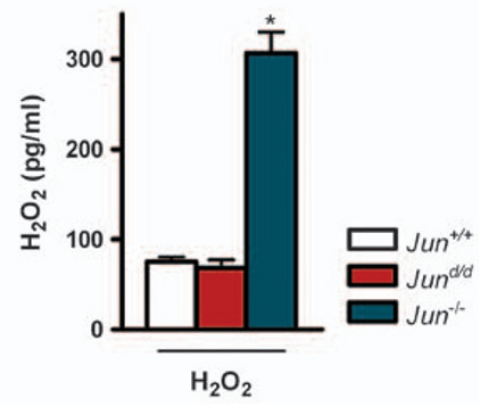

C

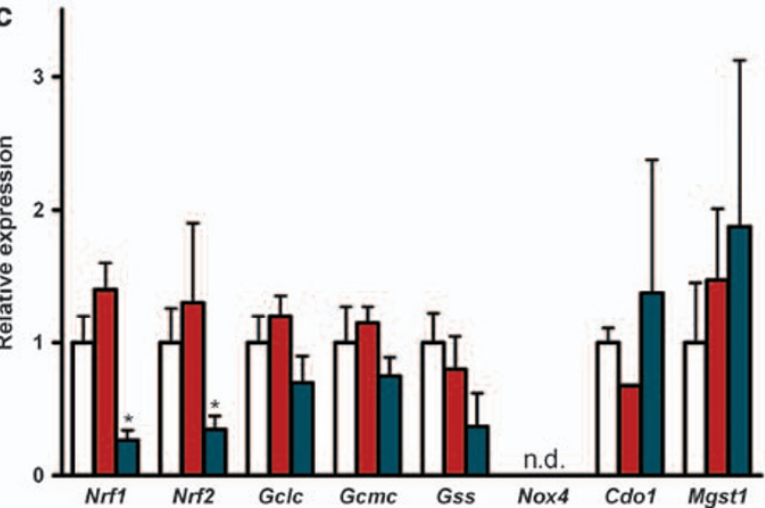

b

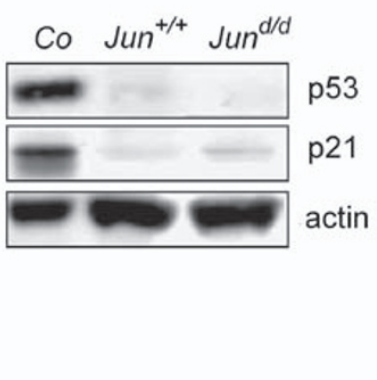

d

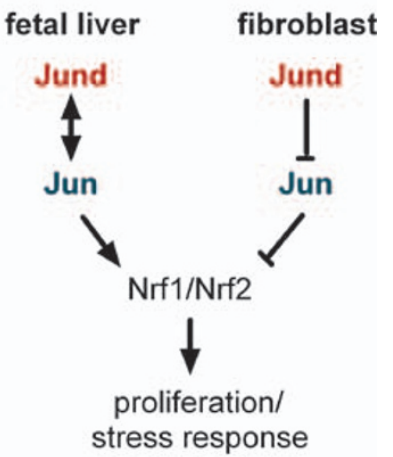

Figure 6 Increased oxidative stress in Jun ${ }^{-1-}$ fetal livers is rescued by JunD. (a) Elevated $\mathrm{H}_{2} \mathrm{O}_{2}$ secretion of $\mathrm{Jun}^{-1-}$ fetal livers is rescued by JunD. $\mathrm{H}_{2} \mathrm{O}_{2}$ levels of freshly isolated fetal livers from Jun ${ }^{+/+}$and Jun ${ }^{-1-}$ mice (lower panel) or Jun ${ }^{+/+}$and Jun ${ }^{d / d}$ (upper panel) were determined by a colorimetric ELISA ( $\left.n=4\right)$. (b) p53 and p21 protein expression in Jun ${ }^{-1-}$ and Jun ${ }^{d / d}$ fetal livers. Co, starved MEF extract; actin serves as loading control. (c) JunD rescues decreased Nrf1 and Nrf2 expression as observed in Jun ${ }^{-1-}$ fetal livers $(n=4)$. The relative mRNA expression of Nrf1/2 and Nrf1/2 targets in primary Jun ${ }^{+/+}$, Jun ${ }^{-1-}$, and Jun ${ }^{d / d}$ fetal livers (qPCR, wild-type set to 1 , $n=4$ ). Error bars represent S.D.; asterisk, $P<0.05$. (d) JunD can substitute for Jun in fetal liver development, but Jun and JunD family members cannot functionally substitute for each other in fibroblast proliferation. The stress response pathway is rescued by JunD, but is dependent on the cellular context

EGFR-dependent signal is necessary for effective activation. A regulatory loop from EGFR signaling to Jun-dependent EGFR expression has been described. ${ }^{34}$ A decrease in EGFR expression and EGFR signaling was observed in Jun ${ }^{\mathrm{d} / \mathrm{d}}$ MEFs and cells lacking Jun. EGFR and Jun have also a prominent function in regulating embryonic eyelid closure. JunD and JunB knock-in mice are born with open eyes, similar to defects observed in mice lacking Jun specifically in the epidermis or disruption of the EGFR. ${ }^{34,36}$ This failure was associated with reduced EGFR expression, supporting an EGFR-dependent signaling pathway to regulate gene expression through AP-1. $J_{u n}{ }^{\mathrm{d} / \mathrm{d}}$ cells responded to EGF or HB-EGF stimulation. In contrast, MEFs lacking Jun did not respond to growth factor treatment, and EGFR and Akt phosphorylation were still impaired. In developing Drosophila eye, low EGFR activity maintains quiescence, similar senescence in which EGF responsiveness and binding is lost and EGFR expression decreases with age. ${ }^{37}$ In addition, reduced EGFR phosphorylation has been observed in senescent cells compared with proliferating cells. ${ }^{38}$ However, the single defect in EGFR expression might not be the main cause of senescence, as MEFs lacking EGFR do not show an obvious proliferation defect (unpublished data). Expression of other EGFR family members was neither affected in Jun ${ }^{-1-}$ fibroblasts nor did pharmacological inhibition of the EGFR pathway interfere with proliferation. EGF treatment did activate p38 activity, which was slightly prolonged in $\mathrm{Jun}^{-1-}$ MEFs. Activation of p38 can also result in premature senescence of MEFs. ${ }^{39}$ Importantly, inhibition of the p38 pathway in $M k k 7^{-1}$ cells rescued these cells from premature senescence. ${ }^{40}$ Thus, a combined effect of defective EGFR signaling, prolonged p38 expression, increased p53 and p21, and reduced CDC2 protein levels may be responsible for the response of $\mathrm{Jun}^{-1-}$ cells to certain growth factors. JunD apparently rescues the endogenous Jun promoter on high EGF treatment through restoration of Jun/AP-1-dependent factors.

Deficiencies in $\mathrm{H}_{2} \mathrm{O}_{2}$ metabolism were observed in Jundeficient fetal livers and MEFs. JunD rescued $\mathrm{H}_{2} \mathrm{O}_{2}$ defects in fetal livers, but not in MEFs. Jun and Nrf1 mutant mice display similar phenotypes, such as embryonic lethality and impaired liver degeneration, ${ }^{27}$ suggesting that these transcription factors may have overlapping but non-redundant functions. On the other hand, JunD and Nrf2 mutant mice are viable, and these transcription factors seem to regulate oxidative stress tolerance and longevity. ${ }^{14,41}$ Thus, Jun and JunD have the ability to protect multiple tissues by regulating detoxification and antioxidant genes as well as cell type-specific targets. However, Nrf1 may be more critical for detoxification, as Nrf2 and expression of other detoxification enzymes is strongly dependent on the genetic background (data not shown).

In summary, JunB and JunD can substitute for Jun in embryonic development. However, Jun functions in fibroblast 
proliferation and senescence are not substituted by JunD. Strong genetic and biochemical evidence supports an antagonistic function for Jun and JunB in cell proliferation. This is likely explained by the differences in the transcriptional activities of Jun and JunB and their respective target genes. On the other hand, JunD is a weaker transactivator, exhibits lower DNA binding, and is atypically expressed. ${ }^{42}$ JunD is likely to bind to different partners than Jun, and differences in dimer composition might, therefore, explain why JunB can rescue proliferation, but not JunD. Moreover, JunD is a JNK substrate, ${ }^{43}$ whereas JunB is not, although impaired $\mathrm{N}$-terminal phosphorylation might not be the reason for incomplete rescue, as Jun ${ }^{A A}$ mice are viable and develop normally. ${ }^{15}$ JunD does differ also from Jun and JunB, as it is not subjected for ubiquitin-mediated degradation. ${ }^{42}$ Thus, prolonged binding and stability of JunD might affect activation or repression of specific target genes. Therefore, Jun, JunB, and JunD are not functionally equivalent and as shown have both overlapping and independent functions. This study identified a novel mechanism how Jun proteins and EGFR signaling control cellular proliferation, cell-cycle arrest/senescence and the response to stress.

\section{Materials and Methods \\ Gene targeting and generation of knock-in mice. The JunD genomic DNA was amplified by PCR, sequence verified, and was cloned into the Jun-mini locus vector. ${ }^{15} \mathrm{R} 1 \mathrm{ES}$ cells were electroporated with $10 \mu \mathrm{g}$ of the Not--linearized knock-in construct and cells were screened for homologous recombination. Four correctly targeted clones out of 1000 analyzed were identified by PCR and Southern analyses as described earlier. ${ }^{15,16}$ Mouse chimeras were generated by injecting correctly targeted ES cell clones into C57/BL6 blastocysts, and germline trans- mission was obtained from two independent clones $(2 \mathrm{H} 4,11 \mathrm{C} 11)$. Heterozygous knock-in mice $\left(\mathrm{Jun}^{\mathrm{d} /+}\right)$ were maintained in a mixed $129(\mathrm{sv}) \times$ C57/BL6 genetic background. Heterozygous mice were then bred to a general Cre-Deleter. Jund ${ }^{-1-}$ mice used in this study have been described earlier. ${ }^{9}$}

Histology. Fetuses and tissues were fixed in $4 \%$ formaldehyde in PBS, embedded in paraffin, $5 \mu \mathrm{m}$ sectioned, and stained either with hematoxylin and eosin according to standard procedures or processed further. Immunohistochemical staining for Ki67 (Novocastra, New Castle, UK) was performed using the ABC staining kit (Vector Laboratories, Burlingame, CA, USA) according to the manufacturer's recommendations. Apoptosis was measured by TUNEL (terminal deoxyribonucleotide transferase-mediated dUTP nick end-labeling staining) using an in situ Cell Death Detection kit (Roche, Indianapolis, IN, USA).

Isolation, growth, and transfection of primary MEFs. MEFs were prepared from wild-type, Jun ${ }^{-/-}$and Jun ${ }^{\mathrm{d} / \mathrm{d}}$ E12.5 fetuses. A total of 2-4 independent MEF lines per genotype were used for the studies. MEFs were cultured in Dulbecco's modified Eagles medium (DMEM) containing 10\% FCS, $2 \mathrm{mM}$ L-glutamine and $100 \mathrm{U} / \mathrm{ml}$ penicillin/streptomycin. Cells at $70 \%$ confluence were arrested by serum deprivation for $48 \mathrm{~h}$ in medium containing $0.5 \%$ serum and stimulated to re-enter cell cycle by adding medium containing $20 \%$ serum or the indicated doses of EGF (Roche). For proliferation assays, MEFs were plated at a density of $3.75 \times 10^{5}$ per $25-\mathrm{cm}^{2}$ flask and passaged every 3-4 days at the same density to determine the cumulative cell number. Senescence $\beta$-galactosidase staining kit protocol was used (Cell Signaling, Danvers, MA, USA). For rescue experiments, embryonic MEFs were seeded at a density of $4 \times 10^{5}$ supplemented with growth factors HB-EGF (Sigma, St Louis, MO, USA) and EGF (Roche) or with antioxidants ascorbate (Vitamin C; Sigma), BHA (Sigma), and NAC (Calbiochem, Darmstadt, Germany) as indicated in the figure. For EGFR inhibitor experiments, fibroblasts were seeded at a density of $1 \times 10^{5}$ per six-well overnight, inhibitors were added the next day, and cells were counted 2 days later. The following EGFR inhibitors were used: EGFR inhibitor (Calbiochem 324674), Erb-B2 inhibitor II (Calbiochem 324732), and EGFR/ErbB-2/ErbB-4 inhibitor (Calbiochem 324840).
The reporter constructs were transfected into Jun ${ }^{+/+}$, Jun ${ }^{\mathrm{d} / \mathrm{d}}$, and Jun ${ }^{-1-}$ MEFs by using the Lipofectamine Plus Reagent (Life Technologies, Carlsbad, CA, USA). Each transfection was performed in triplicate in 24-well plates and the Renilla luciferase reporter pRL-SV40 (Promega, Madison, WI, USA) was cotransfected as an internal control. Promoter luciferase reporter constructs used in the study include p53 (p53-promo-luc) ${ }^{20}$ and cyclin D1 (cD1-promo-luc). ${ }^{24}$ Cells were harvested $24 \mathrm{~h}$ after transfection, or starved in $0.5 \%$ FCS overnight and restimulated for $8 \mathrm{~h}$. Cell extracts were prepared and the Luciferase activity was measured according to the Dual-Luciferase Reporter Assay System (Promega). All Firefly luciferase reporter activities were normalized for transfection efficiency by determining the ratio between Firefly and Renilla luciferase activity. The mean \pm S.D. is shown.

RNA and protein analyses. Total RNA was isolated using Trizol reagent (GIBCO-BRL) or PerfectPure RNA tissue kit (5Prime) and CDNA synthesis was performed as recommended by the Ready-To-Go-You-Prime-First-Strand Beads kit from GE Healthcare (Pittsburgh, PA, USA). PCR amplifications were performed under standard conditions. Primer sequences are available on request. Gene expression of gene markers and controls were analyzed by real-time PCR using BioRad icycler. For each sample, expression of the Hprt, tubulin, or actin gene was used to normalize the amount of the investigated transcript. Protein extracts and western blot analyses were performed according to standard procedures using $20 \mu \mathrm{g}$ of whole cell extracts. The following antibodies were used in this study: anti-JunD antibody (kind gift of D Lallemand; M Yaniv), monoclonal anti-Jun (BD Biosciences, Transduction Lab, San Jose, CA, USA), p53, p21 p16, CDC2, CDK2, CDK4, Cyclin A, Cyclin D1, Cyclin E, phospho-EGFR (Tyr 1173) (all from Santa Cruz, Santa Cruz, CA, USA), phospho-EGFR (Tyr1068), Akt, phospho-Akt, GSK-3 $\beta$, phospho-GSK-3 $\beta$, p42/p44 ERK, phospho-p42/p44-ERK, p38, phospho-p38, JNK, phospho-JNK, EGFR (all from Cell-Signaling), and anti-actin (A2066; Sigma). Proteins were visualized by ECL (Amersham Biosciences (GE Healthcare)).

ChIP experiment. ChIPs were performed as recommended (Milipore, Billerica, MA, USA). The ChIP primers binding in the cyclin D1- and p53-promoter region are described in Supplementary Methods. c-Jun antibody (Santa Cruz; H-79), JunB antibody (Santa Cruz; N-70), JunD antibody (Santa Cruz, 329), and immunoglobulin G (Santa Cruz) were used as unspecific isotype control.

Fetal hepatoblasts cultures. Fetal livers were dissected from wild-type, $\mathrm{Jun}^{-1-}$, and Jun ${ }^{\mathrm{d} / \mathrm{d}} \mathrm{E} 12.5$ fetuses, mechanically dissociated and plated in DMEM containing 10\% FCS, $2 \mathrm{mM}$ glutamine, $1 \%$ penicillin/streptomycin, and $0.1 \mathrm{mM}$ $\beta$-mercaptoethanol. Adherent fetal hepatoblasts were cultivated for 7 days with daily medium change to remove non-adherent hematopoietic cells. At least 2-3 independent fetal livers per genotype were used for the studies.

Hydrogen peroxide colorimetric assay. Fibroblasts or fetal liver cells were cultivated as described and culture supernatants were harvested to measure $\mathrm{H}_{2} \mathrm{O}_{2}$ levels by enzyme-linked immunosorbent assay (Sigma). In brief, particles were removed by centrifugation and diluted supernatant ( $50 \mathrm{mM}$ phosphate, $\mathrm{pH} 6.0$ ) was incubated with a xylenol orange solution in an acidic solution with sorbitol and ammonium iron sulfate that reacts to produce a purple color in proportion to the concentration of $\mathrm{H}_{2} \mathrm{O}_{2}$ in the sample being tested. The optical density was measured after incubation for $30 \mathrm{~min}$ at room temperature using a microplate spectrophotometer set to $550 \mathrm{~nm}$ (SLT, Spectra Image, SLT Labinstruments, Gröding, Austria).

Statistical analyses. Data in bars represent averages \pm S.D. Differences were analyzed by the Student's $t$-test and $P$-values $<0.05$ were considered significant.

\section{Conflict of interest}

The authors declare no conflict of interest.

Acknowledgements. We are very grateful to Drs Latifa Bakiri, Peter Hasselblatt, Maria Sibilia, and John Trichereau for their critical comments and suggestions to the manuscript; Hannes Tkadletz for help in preparing the illustrations. The project was initiated at the IMP, which is funded by Boehringer Ingelheim (BI). JMP and AM are funded by GEN-AU 'Austromouse' grant and IMBA by the Austrian Academy of Sciences. EFW is funded by the BBVA-Foundation and part of the work was also funded by GEN-AU, the IMP, and by grants from Research Training Network (RTN) Program of the European Community, and LK by FWF grant P-18478-B12. 
1. Eferl R, Wagner EF. AP-1: a double-edged sword in tumorigenesis. Nat Rev Cancer 2003 3: 859-868.

2. Shaulian E, Karin M. AP-1 as a regulator of cell life and death. Nat Cell Biol 2002; 4: E131-E136.

3. Hilberg F, Aguzzi A, Howells N, Wagner EF. c-jun is essential for normal mouse development and hepatogenesis. Nature 1993; 365: 179-181.

4. Eferl R, Sibilia M, Hilberg F, Fuchsbichler A, Kufferath I, Guertl B et al. Functions of c-Jun in liver and heart development. J Cell Biol 1999; 145: 1049-1061.

5. Behrens A, Sibilia M, David JP, Mohle-Steinlein U, Tronche F, Schutz G et al. Impaired postnatal hepatocyte proliferation and liver regeneration in mice lacking $\mathrm{c}$-jun in the liver. EMBO J 2002; 21: 1782-1790.

6. Eferl R, Ricci R, Kenner L, Zenz R, David JP, Rath M et al. Liver tumor development. C-Jun antagonizes the proapoptotic activity of p53. Cell 2003; 112: 181-192.

7. Stepniak E, Ricci R, Eferl R, Sumara G, Sumara I, Rath M et al. c-Jun/AP-1 controls liver regeneration by repressing p53/p21 and p38 MAPK activity. Genes Dev 2006; 20: 2306-2314.

8. Hasselblatt $\mathrm{P}$, Rath M, Komnenovic V, Zatloukal K, Wagner EF. Hepatocyte survival in acute hepatitis is due to c-Jun/AP-1-dependent expression of inducible nitric oxide synthase. Proc Natl Acad Sci USA 2007; 104: 17105-17110.

9. Thepot D, Weitzman JB, Barra J, Segretain D, Sinnakre M, Babinet C et al. Targeted disruption of the murine junD gene results in multiple defects in male reproductive system. Development 2000; 127: 143-153.

10. Hilfiker-Kleiner D, Hilfiker A, Kaminski K, Schaefer A, Park JK, Michel K et al. Lack of JunD promotes pressure overload-induced apoptosis, hypertrophic growth, and angiogenesis in the heart. Circulation 2005; 112: 1470-1477.

11. Pillebout E, Weitzman JB, Burtin M, Martino C, Federici $P$, Yaniv M et al. JunD protects against chronic kidney disease by regulating paracrine mitogens. J Clin Invest 2003; 112 843-852.

12. Kawamata A, Izu $Y$, Yokoyama $H$, Amagasa $T$, Wagner EF, Nakashima $\mathrm{K}$ et al. JunD suppresses bone formation and contributes to low bone mass induced by estrogen depletion. J Cell Biochem 2008; 103: 1037-1045.

13. Gerald D, Berra E, Frapart YM, Chan DA, Giaccia AJ, Mansuy D et al. JunD reduces tumor angiogenesis by protecting cells from oxidative stress. Cell 2004; 118: 781-794.

14. Laurent G, Solari F, Mateescu B, Karaca M, Castel J, Bourachot B et al. Oxidative stress contributes to aging by enhancing pancreatic angiogenesis and insulin signaling Cell Metab 2008; 7: 113-124.

15. Behrens A, Sibilia M, Wagner EF. Amino-terminal phosphorylation of c-Jun regulates stress-induced apoptosis and cellular proliferation. Nat Genet 1999; 21: 326-329.

16. Passegue $E$, Jochum W, Behrens A, Ricci R, Wagner EF. JunB can substitute for Jun in mouse development and cell proliferation. Nat Genet 2002; 30: 158-166.

17. Schorpp-Kistner $M$, Wang $Z Q$, Angel $P$, Wagner $E F$. JunB is essential for mammalian placentation. EMBO J 1999; 18: 934-948.

18. Licht AH, Pein OT, Florin L, Hartenstein B, Reuter $H$, Arnold $B$ et al. JunB is required for endothelial cell morphogenesis by regulating core-binding factor $\beta$. J Cell Biol 2006; 175 981-991.

19. Schmidt D, Textor B, Pein OT, Licht AH, Andrecht S, Sator-Schmitt M et al. Critical role for $\mathrm{Nf}-\mathrm{KB}$-induced JunB in VEGF regulation and tumor angiogenesis. EMBO J 2007; 26 710-719.

20. Schreiber M, Kolbus A, Piu F, Szabowski A, Mohle-Steinlein U, Tian J et al. Control of cell cycle progression by c-Jun is p53 dependent. Genes Dev 1999; 13: 607-619.

21. Wisdom R, Johnson RS, Moore C. c-Jun regulates cell cycle progression and apoptosis by distinct mechanisms. EMBO J 1999; 18: 188-197.

22. Shaulian E, Schreiber M, Piu F, Beeche M, Wagner EF, Karin M. The mammalian UV response: c-Jun induction is required for exit from p53-imposed growth arrest. Cell 2000; 103: 897-907.
23. Weitzman JB, Fiette L, Matsuo K, Yaniv M. JunD protects cells from p53-dependent senescence and apoptosis. Mol Cell 2000; 6: 1109-1119

24. Bakiri L, Lallemand D, Bossy-Wetzel E, Yaniv M. Cell cycle-dependent variations in c-Jun and JunB phosphorylation: a role in the control of cyclin D1 expression. EMBO J 2000; 19: 2056-2068.

25. Pfarr C, Mechta F, Spyrou G, Lallemand D, Carillo S, Yaniv M. Mouse JunD negatively regulates fibroblast growth and antagonizes transformation by ras. Cell 1994; 76: 747-760.

26. Meixner A, Karreth F, Kenner L, Wagner EF. JunD regulates lymphocyte proliferation and $T$ helper cell cytokine expression. EMBO $j 2004 ; 23: 1325-1335$.

27. Chen $L$, Kwong $M$, Lu R, Ginzinger $D$, Lee $C$, Leung $L$ et al. Nrf1 is critical for redox balance and survival of liver cells during developlment. Mol Cell Biol 2003; 23: 4673-4686.

28. Chan K, Lu R, Chang JC, Kan YW. NRF2, a member of the NFE2 family of transcription factors, is not essential for murine erythropoiesis, growth, and development. PNAS 1996; 93: $13943-13948$.

29. Reddy NM, Kleeberger SR, Bream JH, Fallon PG, Kensler TW, Yamamoto M et al. Genetic disruption of the Nrf2 compromises cell-cycle progression by impairing GSH-induced redox signaling. Oncogene 2008; 27: 5821-5832.

30. Yang $\mathrm{H}$, Magilnick $\mathrm{N}$, Lee $\mathrm{C}$, Kalmaz D, Ou X, Chan JY et al. Nrf1 and Nrf2 regulate rat glutamate-cysteine ligase catalytic subunit transcription indirectly via NF-kappaB and AP-1. Mol Cell Biol 2005; 25: 5933-5946.

31. Beyer TA, Xu W, Teupser D, auf dem Keller U, Bugnon P, Hildt E et al. Impaired liver regeneration in Nrf2 knockout mice: role of ROS-mediated insulin/IGF-1 resistance. EMBO J 2008; 27: 212-223.

32. Lee JM, Li J, Johnson DA, Stein TD, Kraft AD, Calkins MJ et al. Nrf2, a multi-organ protector? FASEB J 2005; 19: 1061-1066.

33. Eto I. Molecular cloning and sequence analysis of the promoter region of mouse cyclin D1 gene: implication in phorbol ester-induced tumour promotion. Cell Prolif 2000; 33: 167-187.

34. Zenz R, Scheuch $\mathrm{H}$, Martin $\mathrm{P}$, Frank $\mathrm{C}$, Eferl $\mathrm{R}$, Kenner $\mathrm{L}$ et al. c-Jun regulates eyelid closure and skin tumor development through EGFR signaling. Dev Cell 2003; 4: 879-889.

35. Sekhar KR, Meredith MJ, Kerr LD, Soltaninassab SR, Spitz DR, Xu ZQ et al. Expression of glutathione and gamma-glutamylcysteine synthetase mRNA is Jun dependent. Biochem Biophys Res Commun 1997; 234: 588-593.

36. Sibilia M, Wagner EF. Strain-dependent epithelial defects in mice lacking the EGF receptor. Science 1995; 269: 234-238.

37. Yang $\mathrm{L}$, Baker NE. Cell cycle withdrawal, progression, and cell survival regulation by EGFR and its effectors in the differentiating Drosophila eye. Dev Cell 2003; 4: 359-369.

38. Brooks KM, Phillips PD, Carlin CR, Knowles BB, Cristofalo VJ. EGF-dependent phosphorylation of the EGF receptor in plasma membranes isolated from young and senescent WI-38 cells. J Cell Physiol 1987; 133: 523-531.

39. Wagner EF, Nebreda AR. Signal integration by JNK and p38 MAPK pathways in cancer development. Nat Rev Cancer 2009; 9: 537-549.

40. Wada T, Stepniak E, Hui L, Leibbrandt A, Katada T, Nishina H et al. Antagonistic control of cell fates by JNK and p38-MAPK signaling. Cell Death Differ 2008; 15: 89-93.

41. Motohashi H, Yamamoto M. Nrf2-Keap1 defines a physiologically important stress response mechanism. Trends Mol Med 2004; 10: 549-557.

42. Hernandez JM, Floyd DH, Weilbaecher KN, Green PL, Boris-Lawrie K. Multiple facets of junD gene expression are atypical among AP-1 family members. Oncogene 2008; 27: 4757-4767.

43. Gupta S, Barrett T, Whitmarsh AJ, Cavanagh J, Sluss HK, Dérijard B et al. Selective interaction of JNK protein kinase isoforms with transcription factors. EMBO J 1996; 15: $2760-2770$.

Supplementary Information accompanies the paper on Cell Death and Differentiation website (http://www.nature.com/cdd) 